\title{
It's a Matter of Time
}

\section{Robert A. Barrett Jr.}

Honolulu, HI, USA

Email: rbjrpa@gmail.com

Received 7 March 2015; accepted 15 May 2015; published 19 May 2015

Copyright (C) 2015 by author and Scientific Research Publishing Inc.

This work is licensed under the Creative Commons Attribution International License (CC BY).

http://creativecommons.org/licenses/by/4.0/

(c) (i) Open Access

\section{Abstract}

This paper describes a universe consisting only of time dilation and compares it to the one which we observe.

\section{Keywords}

Time Dilation, Gravity, Light, Electricity, Magnetism

\section{Introduction}

I would first like to apologize that this paper may not be up to the rigor that you are accustomed to. At best, I consider myself an amateur with a penchant for out-of-the-box thinking. That said I have a perspective that is important to share.

Matter has been described with numerous characteristics that almost appear magical at times such as gravity, atomic repulsion, etc., but possibly the most intriguing is that it dilates time. This time dilation is seen as an outcome of gravity and the constant speed of light. I would like to take a moment to discuss the opposite, a world where there are only dilations in time and we observe matter and energy as the interactions of these dilations.

\section{Time Points}

If we formulate the time dilation similar to the Schwarzschild formulation [1], substituting the mass and gravitational multiplier with 1 , it would appear as the following equation. Time would slow as the distance to the location of the time dilation decreased. That which would take a fraction of a second in real time could take billions of years in relative time as shown in Figure 1.

$$
\frac{\mathrm{d} t^{\prime}}{\mathrm{d} t}=\sqrt{1-\frac{1}{h T}}
$$




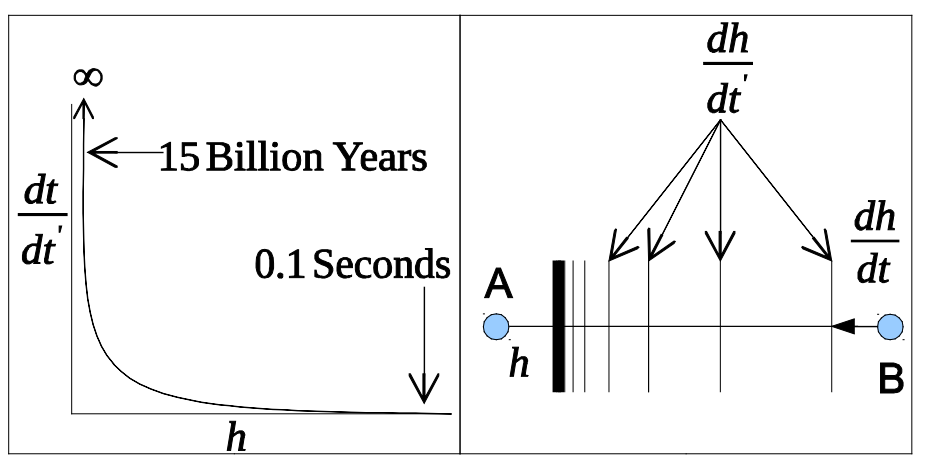

Figure 1. The dilation of time is asymptotic with its reciprocal approaching infinity as distance decreases. A constant movement in real time results in slower movement in relative time.

where $t^{\prime}$ is the location specific relative time as observed by someone unaffected by the time dilation; $t$ is the real time that takes place absent of any dilation; $h$ is the distance from the time dilation; $T$ is the time dilation coefficient.

This is an asymptotic relationship showing that the dilation in time approaches $1 / \infty$ with less and less distance. This indeed describes more of a point and not an object with physical dimensions. Its main dimensionality becomes its impact on time around it. In such a universe of relative time, these points of time dilation or "time points" would be all that exists.

Their impact across distances would be faint and not observable. A single time point could only be observed when another time point were to come within a distance that causes enough of an impact for the observer to witness. These time points would remain unobservable while distances between them are substantial.

As two time points, A and B, move in close proximity towards one another, relative time for each would change. As B approaches A, time would slow. Any given movement under real time would translate into a slower movement in relative time. B would never be able to occupy the same location as A because time would slow to infinity before they could move to overlap: $h \downarrow, \mathrm{d} h / \mathrm{d} t^{\prime} \downarrow \downarrow$.

Mathematically, this can be represented by the following set of equations. As time point B moves towards A, it would initially be travelling along vector $V_{B}$, where, treating it as an array, $V_{B}=(\mathrm{d} x, \mathrm{~d} y, \mathrm{~d} z)$ in real time while in relative time, $V_{B}^{\prime}=V_{B}\left(\frac{\mathrm{d} t^{\prime}}{\mathrm{d} t}\right)$ or $V_{B}^{\prime}=V_{B}\left[\frac{\partial t^{\prime}}{\partial t}+\frac{\partial t^{\prime}}{\partial h} \frac{\partial h}{\partial x}, \frac{\partial t^{\prime}}{\partial t}+\frac{\partial t^{\prime}}{\partial h} \frac{\partial h}{\partial y}, \frac{\partial t^{\prime}}{\partial t}+\frac{\partial t^{\prime}}{\partial h} \frac{\partial h}{\partial z}\right]^{\mathrm{T}}$. Comparing vector $V_{B}^{\prime}$, the movement in relative time, with vector $V_{B}$ we see that the components that would make them differ in direction are $\partial h / \partial x, \partial h / \partial y$, and $\partial h / \partial z$ which can also be defined as $\cos \left(\theta_{A B x}\right), \cos \left(\theta_{A B y}\right)$, and $\cos \left(\theta_{A B z}\right)$ respectively. The impact that time dilation would have can best be demonstrated when we define our coordinate system so that the two time points are both on the $x$ axis. There, $\cos \left(\theta_{A B x}\right)=-1, \cos \left(\theta_{A B y}\right)=0$, and $z=0$ causing the time dilated vector to be $V_{B}^{\prime}=V_{B}\left[\frac{\partial t^{\prime}}{\partial t}-\frac{\partial t^{\prime}}{\partial h}, \frac{\partial t^{\prime}}{\partial t}, 0\right]^{T}$ demonstrating that movement in relative time is not the same as real time. One could translate this vector in relative time back to a new vector in real time resulting in a new vector in real time with a new $\mathrm{d} x$ subsequently changing the trajectory.

An alternative interpretation is that as time point B approaches $A$ as in Figure 2, the movement towards time point A slows while the movement perpendicular to time point A does not, allowing time point $\mathrm{B}$ to move slightly more along its tangent.

The motion of time point B towards time point A is relative. As B approaches A, A approaches B. In this way, the vector $V_{B}$ has a reciprocal vector $V_{A}$ that has an equal but opposite motion. Because of the increased movement in relative time along the opposite tangents, time points $\mathrm{A}$ and $\mathrm{B}$ would rotate around one another in relative time, when compared to other time points at a distance.

Eventually, the two time points would pass one another in real time and $V_{B}$ would begin to increase the distance between time points A and B. When this happens the impact of time dilation would dissipate until it is indistinguishable to any other time point. 


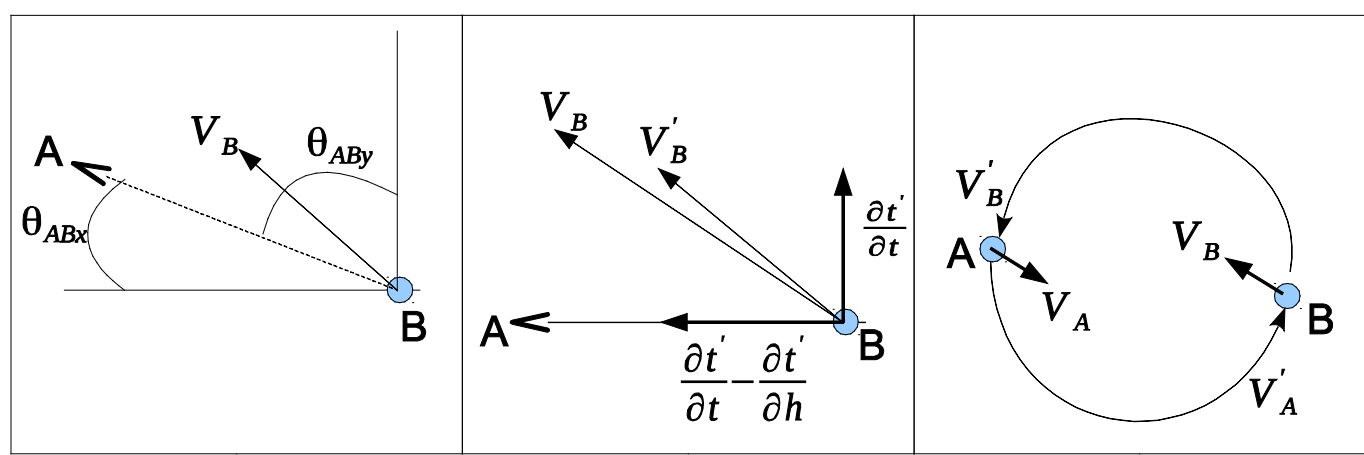

Figure 2. As time points approach one another, a set trajectory in real time would be seen as a changing trajectory in relative time and would present itself as a rotation to external elements.

A third time point could also come near and disrupt this interaction between $\mathrm{A}$ and $\mathrm{B}$. Time point $\mathrm{C}$ could dilate time more for time point B than for time point A and alter B's trajectory relative to A. This could break the relationship of $\mathrm{AB}$ apart even before the movement of the original vectors could. For these reasons, the interactions between two time points would be temporary and not a stable construct.

A third time point, C, however, could encounter time points A and B in a specific way, resulting in a more interesting relationship. Assuming that time dilation is additive, we can represent the vector $V_{C}^{\prime}$ as follows,

$$
\begin{aligned}
& \alpha_{C}=\frac{\partial t_{C A}^{\prime}}{\partial t}+\frac{\partial t_{C B}^{\prime}}{\partial t}, \quad \beta_{C A j}=\frac{\partial t_{C A}^{\prime}}{\partial h_{C A}} \frac{\partial h_{C A}}{\partial j}, \quad \beta_{C B j}=\frac{\partial t_{C B}^{\prime}}{\partial h_{C B}} \frac{\partial h_{C B}}{\partial j}, \\
& V_{C}^{\prime}=V_{C}\left[\alpha_{C}+\beta_{C A x}+\beta_{C B x}, \alpha_{C}+\beta_{C A y}+\beta_{C B y}, \alpha_{C}+\beta_{C A z}+\beta_{C B z}\right]^{\mathrm{T}} .
\end{aligned}
$$

where $\alpha$ is the static time dilation based on a time point's position relative to other time points being examined; $\beta$ is the tangential time dilation that is occurring due to the movements of the various time points being considered; $j$ represents the $x, y, z$ axis.

We can examine the individual $\beta \mathrm{s}$ and see that there are situations where the trajectory of $V_{C}^{\prime}$ would be altered in such a way as to move towards the center of time points $\mathrm{A}$ and $\mathrm{B}$. When $\beta_{C A j}$ and $\beta_{C B j}$ are of opposite signs, the corresponding trajectory would continue to travel between time points $A$ and $B$. Even in three dimensional space, as a slower moving time point, $\mathrm{C}$, moves towards the circle produced by a rotating $\mathrm{A}$ and $\mathrm{B}$, we will see $\beta_{C A j}$ and $\beta_{C B j}$ remaining with opposite signs

More important though, is that when, as in Figure 3, time point $\mathrm{C}$ is equidistant to $\mathrm{A}$ and $\mathrm{B}, h_{C A}=h_{C B}$, then $\beta_{C A j}=\beta_{C B j}$. When this happens $V_{C}^{\prime}$ would continue on the same slope as $V_{C}$. Eventually, the distances between all three time points will become indistinguishable from one another eventually reaching the distance, $\delta$, at which, for the sake of any other interactions, time can be considered to stop. This results in the situation where all three time points are in equilibrium. This creates the first stable set of time points. One that could not be pulled apart by themselves or by other sets of time points merely coming into their vicinity.

As another time point $\mathrm{D}$ would near the set of time points $\mathrm{ABC}$, it would be slowed based on the combined time dilations of ABC and prevented from moving towards them. However, the time dilation caused by D would not be enough to prevent the set $\mathrm{ABC}$ to move towards it.

$$
\frac{\mathrm{d} t}{\mathrm{~d} t_{A D}^{\prime}}+\frac{\mathrm{d} t}{\mathrm{~d} t_{B D}^{\prime}}+\frac{\mathrm{d} t}{\mathrm{~d} t_{C D}^{\prime}}>\frac{\mathrm{d} t}{\mathrm{~d} t_{D A}^{\prime}}, \frac{\mathrm{d} t}{\mathrm{~d} t_{D B}^{\prime}} \text {, and } \frac{\mathrm{d} t}{\mathrm{~d} t_{D C}^{\prime}}
$$

External observers would see $\mathrm{D}$ being swept along with time points $\mathrm{ABC}$ until its vector starts to move away from $\mathrm{ABC}$ or it encounters other time point(s) that would release it by altering time point D's vector relative to $\mathrm{ABC}$. The set of time points $\mathrm{ABC}$ would eventually approach the distance, $\delta$ from one another essentially forming the points of an equilateral triangle.

Such a stable geometry would have another distinct feature. It is progressive. In other words, it can come together while A and B are already interacting with one another. Other stable geometries may be constructed, but with the exception of the next two, they require time points aligning themselves in a more simultaneous fashion. 
The next progressive and stable geometry, would require the fourth time point $\mathrm{D}$ to travel in three dimensional space on a vector that takes it through the triangle formed by time points ABC. If one looks at the triangular set of time points in three dimensions, one will find that there is a location directly above and below its center that is equal distance from all three time points. A time point heading through the triangle would have $\beta \mathrm{s}$ that would move it into this location where it would find that it has similar time dilations to all three time points in the approaching triangle. At such a location, time point $\mathrm{D}$, though still moving towards the center would have its tangential movement equalized by the other time points. This is depicted in Figure 4 .

This equilateral triangular pyramid of time points would also be stable. Other time points would not be able to unlock any of the four time points in such a structure. Other time points could approach this pyramid but none would be immune to other sets of time points moving them away. Also, because it is formed by adding a single time point to an existing set, the triangle, it is progressive and easily formed.

A third stable and progressive geometry would also arise if two stable triangles, as in Figure 5, were to align with their centers moving through the other's triangle. As this trajectory continues to be altered in relative time, the planes of the triangles would become parallel and the time points would become rotated 60 degrees from one another. The resulting relationship would have six points with eight equilateral triangles providing eight planes or sides. Once again, this geometry would be stable in that no other set of time points could influence one time point in the structure independent of the others and although an external time point would be prevented in moving closer to this group, the group would not be stopped by them.

In such a universe of relative time, these time points and their subsequent geometries would make up the foundations of matter and energy while moving time dilators are the only thing that exists. An example of such stable geometries is given in Table 1.

Time points that are not in relationships with other time points would act like dark matter whose presence in large numbers may be felt while remaining individually elusive. The unstable relationships of pairs or other unstable combinations, with their short existence, would present themselves similar to quarks. The first stable

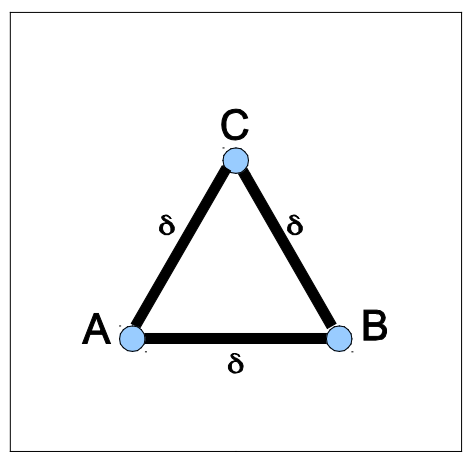

Figure 3. As time point $\mathrm{C}$ moves into an equilateral position, an equilibrium is reached, locking the three time points together.

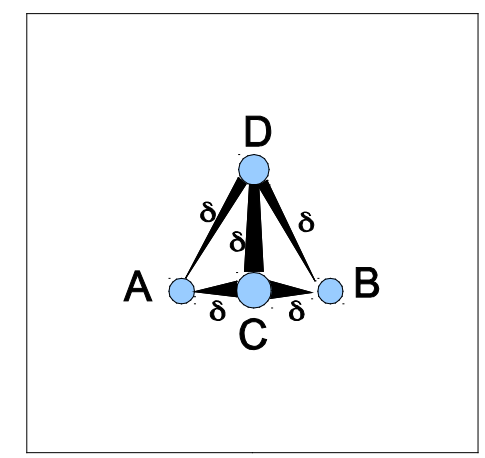

Figure 4. A second progressive geometry is obtained when a forth time point moves into an equilateral position forming an equilibrium among the four time points. 


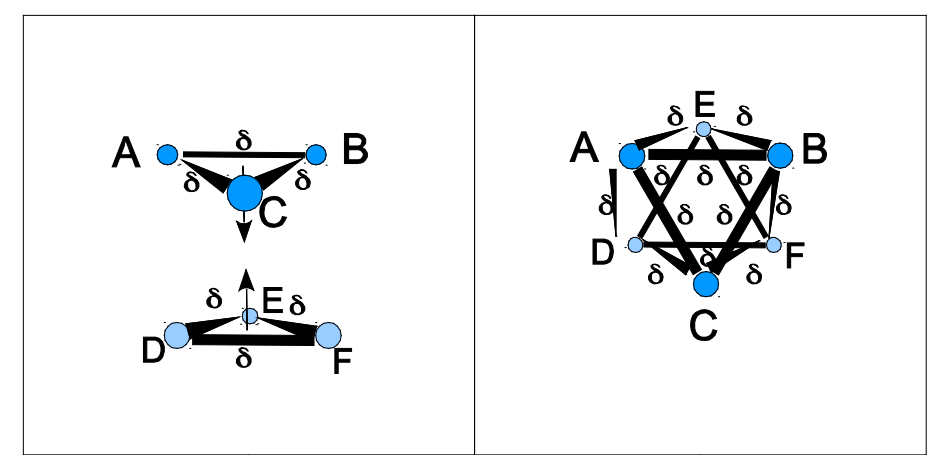

Figure 5. As two triangles as in Figure 3 come together, a new equilibrium is obtained creating the third geometric set of time points.

Table 1. Progressive stable geometric sets.
\begin{tabular}{ccccccccccc} 
Time Point & \multicolumn{1}{c}{ First } \\
\cline { 2 - 10 }$(\delta$ Units $)$ & $x$ & $y$ & $z$ & $x$ & $y$ & $z$ & $x$ & $y$ & $z$ \\
\hline A & -0.5 & -0.2887 & 0.0 & -0.5 & -0.2887 & -0.1443 & -0.5 & 0.2887 & -0.1443 \\
B & 0.5 & -0.2887 & 0.0 & 0.5 & -0.2887 & -0.1443 & 0.5 & 0.2887 & -0.1443 \\
C & 0.0 & 0.5773 & 0.0 & 0.0 & 0.5773 & -0.1443 & 0.0 & -0.5773 & -0.1443 \\
D & & & & 0.0 & 0.0 & 0.1443 & -0.5 & -0.2887 & 0.1443 \\
E & & & & & & & 0.0 & 0.5773 & 0.1443 \\
F & & & & & & & 0.5 & -0.2887 & 0.1443 \\
\hline
\end{tabular}

construct, the equilateral triangles would be the smallest of the stable constructs and represent electrons. The equilateral pyramid would be only slightly larger than the electron and act as a neutron. The largest, the proton, would be the eight sided equilateral and because of its increased number of time points, it would dominate the others.

As these subatomic constructs move about, their interactions would be driven by the same principals of time dilations that formed them. As neutrons move towards protons, and vice versa, time would dilate joining them by making this movement take forever. Electrons would join with such a nucleus in a similar fashion. Multiple protons and neutrons, under the right circumstances, would obtain vectors that keep them associated with one another.

\section{Forces Are Phenomena}

As these basic structures making up a universe of time dilation move through three dimensional space, their interactions with other groups of time points would cause specific movements that would appear as separate forces. The first obvious phenomenon that would appear as a force is the phenomenon of time slowing as sets of time points approach one another. As sets of time points approach one another, the time dilation increases to infinity. As the resulting movement of the set slows, it would give the appearance that the one set is repelling the other. That which would be described as atomic repulsion would be merely time dilating to its extreme as objects approach one another and the resulting tangential movement that is accompanied with it.

Other phenomena would be caused by the varied distances of each of the time points in an equilibrium. The time points of an electron, neutron, or proton, would be at different distances to other sources of time dilation and therefore have different relative time. Though tied to one another by their relative motions to one another, the individual time points would move at different rates when confronting separate sources of time dilation. These unequal movements would be identified as separate forces but actually be phenomena caused by time dilation.

Relative to a separate group of time points, three distinct movements can be characterized for any two time points in an equilibrium. The first is the movement towards one another. As the slope of the time dilation function approaches perpendicular as the distance decreases, the differences in time dilation between the closer time 
point and the further can be significant. Even at the distance $\delta$ the time points are still moving towards one another. As the two time points move extremely close to another source of time dilation, the further time point would move faster than the closer time point causing the pair to move closer to the separate source.

Another movement would be the pair of time points in equilibrium moving past the separate mass of time points. As discussed, the equilibriums of time points in a time dilation universe would be at different distances and any pair that is not equal distant to the separate mass would have the closer time point moving past the mass at a slower rate than the further time point.

If we make the simplifying assumption that the separate mass, $M$, can be described as a collection of $N$ time points whose combined effect is equivalent to their average distance to each of the time points contained in the equilibrium set of time points, we can state the time dilation as follows,

$$
h_{j}=\frac{\sum h_{j i}}{N}, \frac{\mathrm{d} t_{j}^{\prime}}{\mathrm{d} t} \approx \sqrt{1-\frac{N}{h_{j} T}}
$$

where $h_{j i}$ the distance of time point $j$, within the equilibrium, from time point $i$, within the mass; $h_{j}$ the distance of time point $j$, within the equilibrium, from the center of the mass; $N$ is the number of time points within the mass.

At great distances the hyperbolic time dilation function takes on linear characteristics. At these distances, the differences in time dilation between the individual time points become small, therefore any phenomenon caused by the distant source of time dilation also becomes insignificant.

As the distances become smaller, though, the hyperbolic nature of time dilation dictate that the differences in relative time between the individual time points, increase. Eventually these differences in relative time will become dramatic enough to alter the behavior of the set. Absent any separate source of time dilations, two time points in equilibrium would mimic the movement of a stick. As depicted in Figure 6, the two ends would travel together moving them in the same direction. If one end moves faster than the other, the two ends would circle around a mid position.

For any two time points locked in an equilibrium, the farthest will always move faster than the nearer time point when they are close to a source of time dilation when viewed in relative time. This results in the third movement, a pair circling a mid position. We can isolate this circular motion by defining the circular motion, $V_{c f}$, for the far time point and $V_{c n}$, for the near time point as follows,

$$
\begin{aligned}
& V_{c f}=\left(\left(\mathrm{d} x_{f}-\mathrm{d} x_{n}\right) / 2,\left(\mathrm{~d} y_{f}-\mathrm{d} y_{n}\right) / 2,\left(\mathrm{~d} z_{f}-\mathrm{d} z_{n}\right) / 2\right) \\
& V_{c n}=\left(\left(\mathrm{d} x_{n}-\mathrm{d} x_{f}\right) / 2,\left(\mathrm{~d} y_{n}-\mathrm{d} y_{f}\right) / 2,\left(\mathrm{~d} z_{n}-\mathrm{d} z_{f}\right) / 2\right)
\end{aligned}
$$

Given that the two time points must remain in equilibrium, then $V_{c f}^{\prime}+V_{c n}^{\prime}=0$. but because the two time points are at different distances to the source of time dilation, $V_{c f}\left(\mathrm{~d} t_{f}^{\prime} / \mathrm{d} t\right)^{c}>-V_{c n}\left(\mathrm{~d} t_{n}^{\prime} / \mathrm{d} t\right)$ an additional movement is required to maintain this equilibrium: $V_{c f}\left(\mathrm{~d} t_{f}^{\prime} / \mathrm{d} t\right)+V_{c n}\left(\mathrm{~d} t_{n}^{\prime} / \mathrm{d} t\right)+V_{c m}^{\prime}=0$. Here, $V_{c m}^{\prime}$, is the movement of the overall pair in relative time.

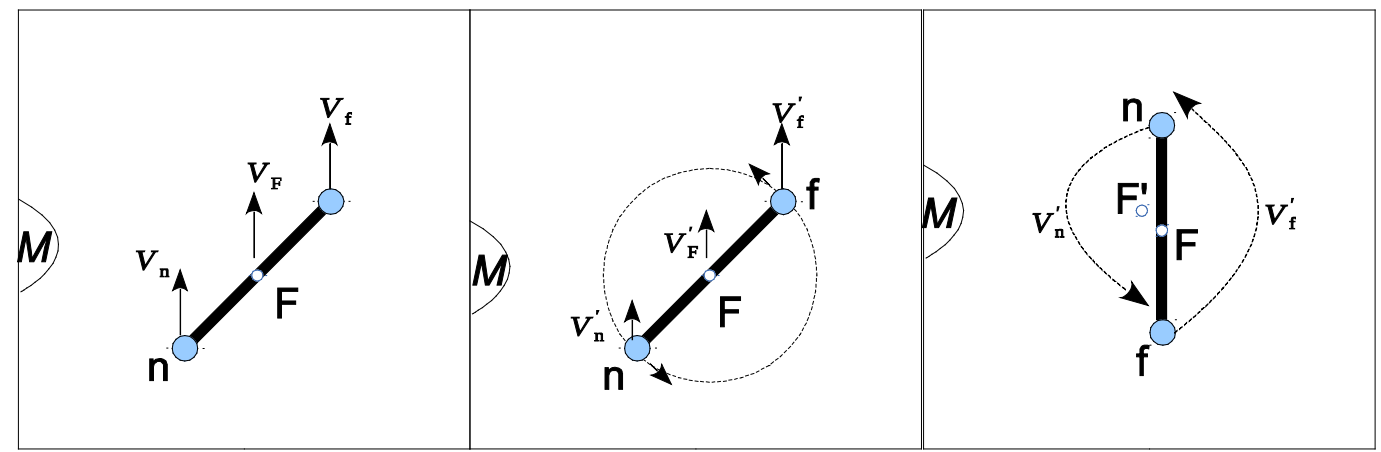

Figure 6. A pair of time points in equilibrium would travel together in real time but in relative time they will circle, shifting their fulcrum in the direction of the circle of the far time point and towards the slower spinning time point or the distant mass. 
Examining, $V_{c m}^{\prime}$, we can determine that this will be consistently in the direction that the far time point is circling and on the side of the source of time dilation. This is illustrated in Figure 6 where the dynamics are similar to a lever. The distance traveled by the far time point will be larger in relative time than that of the near time point causing the fulcrum of the two points to shift towards the mass. The new movements of these time points can be translated back to real time causing the shifted fulcrum in relative time to become the mid position in real time. This happens while the time point circles half a revolution around the mid position. Upon completion of half a revolution, when both time points become equidistant to the mass, the roles will reverse and the time point that was near becomes the far time point and the far becomes the near. This continues to shift the fulcrum towards the source of time dilation.

Along with this, the pair travels further in the direction that the far time point is circling and shorter in the direction of the near time point. This moves the overall pair in that direction in relative time. When this is translated back to real time this adds extra distance to the original vector causing the pair to increase their speed in that direction. Also, the faster the circular motion, the bigger this lateral motion will be.

As the two time points get closer and closer to a separate source of time dilation, the differences in the vectors increase. This causes the movement towards the mass to increase and the movement in the direction of the far time point to increase. This may be observed as a slow moving set because the overall time dilation is so high while the actual speed in real time is extreme.

As demonstrated, these movements are tied together. Each of the subatomic constructs in a world of time dilation would have multiple pairs of time points moving about and, with the exception of when time points are equidistant, they will all be combining to move the construct towards the other source of time dilation. Movement within the equilibrium, therefore, impacts the circular motion and lateral movement, circular motion also impacts lateral movement and changes internal movement, and lateral movement would impact circular motion and internal movement. It is only knowing each movement will the total phenomenon be really understood.

Each of the equilibrium structures, the electron, neutron, and proton, would have this distinctive attractive phenomena while each would also have a distinctive repulsion phenomena based on the exponential slowing of time. These would be minor when viewed individually at large distances from a mass. When combined, however, the phenomena can be much more dramatic.

As a neutron (or electron) is drawn to a proton, it's forward movement will slow while it's lateral movement will increase dramatically. This means the neutron and proton, relative to a distant mass, will be circling very fast around a focal position when they reach their own $\delta$ distance where, for all purposes, they cannot get meaningfully closer. This puts the neutron and proton in their own equilibrium, forming a nucleus.

As seen in Figure 7, connecting the time points within the neutron with the time points within the proton produces 24 pairs of time points in equilibrium with one another. Their average distance will be the distance between the mid position of the proton and the mid position of the neutron. This average distance will be considerably further apart than the distances within any of the subatomic equilibriums. An electron moving towards a combined neutron and proton, will also result in additional pairings, extremely fast circling, along with greater equilibrium distances.

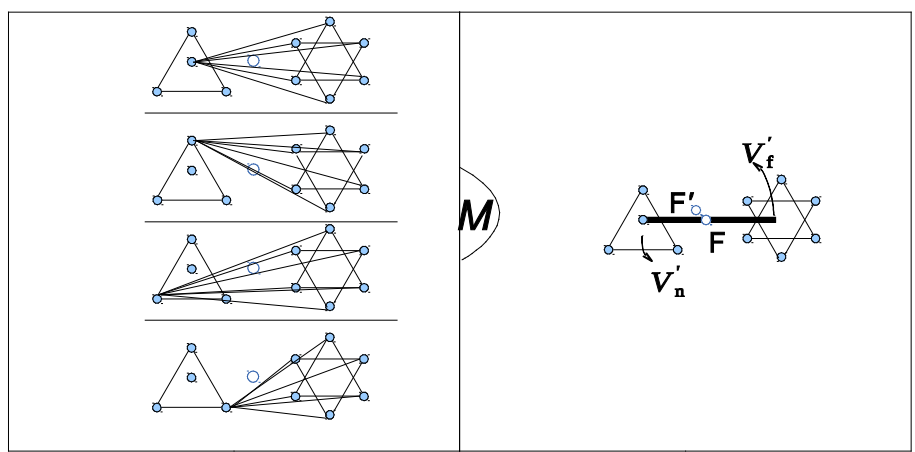

Figure 7. A neutron and proton would circle extremely fast around one another with 24 pairs of time points in equilibrium with a much greater distance between them resulting in significant changes in their fulcrum position towards a distant source of time dilation. This would appear as the unseen force we observe as gravity. 
As the three components, proton, neutron, and electron, are drawn together in a universe of time dilation, they form the basic atomic structure that we are familiar with. Because the extreme circling speed caused by the lateral motion within the atomic structure is combined with the larger distances between time points in the atom and the multitude of rotating pairs, the resulting movement towards the mass would be most significant. This would be observed as gravity but instead of being an unseen force, it is merely the phenomena of circling subatomic pieces.

This also implies that gravity is created by the combining of subatomic parts while individually they would show a much smaller movement towards a source of time dilation. In other words, a neutron will appear to have very little mass until it is circling a proton causing a much greater attraction. This also indicates that gravity is a function of the number of time points, the time dilation coefficient, and the distance at which forward movement is essentially stopped: $G=f(T, \delta, N)$.

As the various structures move about in such a universe, they are themselves governed by the same properties of time dilation that brought them together. As a proton moves towards other protons, their momentums will be blocked by time dilation, fusing them together. Neutrons and electrons could then move towards this increased source of time dilation forming different elements. In-turn, these atomic structures would move towards other atomic structures resulting in similar interactions governed by the varied time dilations of each of the component time points. As time dilates towards infinity, these atoms would become locked in the form associated with molecules, until some other source of time dilation were to impact them and change the trajectories of an individual atom.

\section{Two Dimensional Electrons: Movement and Rotation}

Electrons in a universe of time dilation would have strange and "funky" behavior similar to our own universe. This can be attributed to being a two dimensional equilibrium of time points in three dimensional space. The movements of an electron relative to a distant mass, as shown in Figure 8, can be broken into three separate movements. The basic movement is the overall movement of the mid position of the equilibrium, $V_{m}$. Another can be described as the spin, $V_{s}$, which would be the movement around the plane generated by the three time points. A final movement can be identified as the rotation of the plane, $V_{R}$, around the mid position relative to the distant mass. These vectors could be represented as follows,

$$
V_{m}=\left(\mathrm{d} x_{m}, \mathrm{~d} y_{m}, \mathrm{~d} z_{m}\right), V_{s}=\left(\mathrm{d} \theta_{s x}, \mathrm{~d} \theta_{s y}, \mathrm{~d} \theta_{s z}\right), V_{R}=\left(\mathrm{d} \theta_{R x}, \mathrm{~d} \theta_{R y}, \mathrm{~d} \theta_{R z}\right)
$$

where $\theta_{s x}$ the angle of $\angle \mathrm{A} m x$, time point A to the mid position, $m$, to the $x$ axis, etc.; $\theta_{R x}$ the angle of the plane to the $x$ axis, etc.

As an electron moves amongst other constructs, it would interact with these constructs based upon the changes in time dilation as time points approach one another and move away from each other. This interaction would change the directions of the electrons and the atoms they are interacting with, causing them to in-turn change directions. This increased movement could also cause atoms that are not associated with one another to travel towards each other potentially locking one another together. As atoms move on vectors that take them

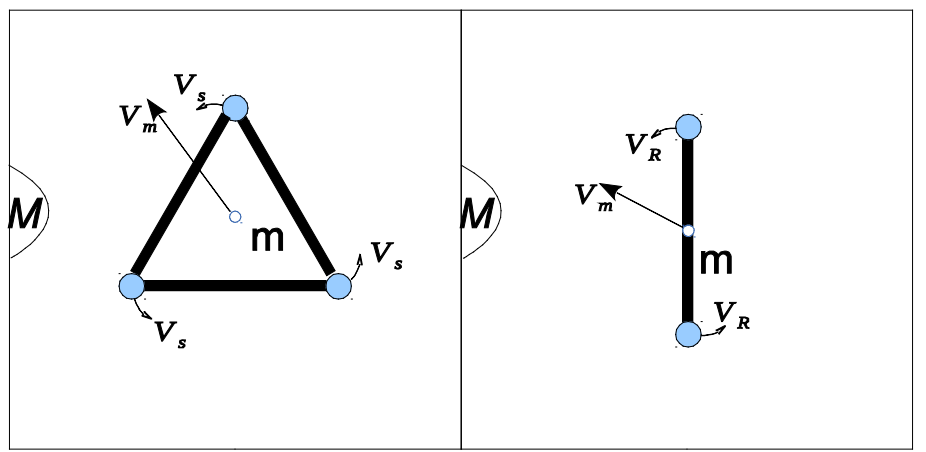

Figure 8. A two dimensional electron would have three major motions, movement of the mid position $m$, spin around its plane, $s$, and rotation of the plane, $R$. 
through one another, time would dilate to infinity keeping them bound to one another. Likewise, atoms that are currently locked because they are moving towards one another could have their directions changed resulting in them unlocking from one another. This movement would be perceived as heat is perceived.

As the mid position of an electron moves, the rotation around the mid position relative to a separate mass would exhibit the characteristics of a wave. As the electron rotates, it flips end over end like a coin being tossed. From a distant mass, the distance between the closest time point to the furthest varies as the electron rotates. As the distance varies, so does relative time, causing the speed that each time point travels to be different.

The difference in relative time on the rotating electron and to a lesser extent, the time dilation that the electron has on other constructs would increase and decrease in accordance with the angle of the rotation with respect to the direction the electron is traveling. This effect would result in a typical sine wave as the electron rotates half a rotation around its mid position. The distance that the electron's mid position travels as it makes this half rotation would be equivalent to its wavelength resulting in Equation (4).

$$
\lambda=V_{m}^{\prime}\left(\pi / V_{R}^{\prime}\right)
$$

where $\lambda$ is the wavelength; $\pi / V_{R}^{\prime}$ is the amount of time the electron takes to make half a revolution.

As the plane of the electron is aligned with a mass, the time point closest will have the slowest movement while the furthest time point will have the fastest and because of the exponential property of time dilation, the differences would be negative when viewed from a distant mass.

Again, the fulcrum position would change towards the distant mass. This would cause the overall path of the electron to change in relative time towards the mass. This is the behavior of a gravitational lens. This phenomenon would also be exaggerated as the distance to the mass becomes increasingly small. As a rotating electron comes extremely close to a group of atoms in real time, the differences between the movement of the far time point and the near time point would substantially increase and in relative time, the electron would bend towards the atoms. This describes diffusion.

Furthermore, the amount of bending would be a result of the orientation of the rotating electron as it passes. As in Figure 9, an electron passing as its rotation is aligned with the mass will have its course changed more than one that is at a 45 degree angle as it passes. An electron whose plane is parallel to the mass as it passes, would pass straight by this mass. Given an appropriate structure, electrons traveling with different wavelengths will pass the atoms composing the structure with different orientations resulting in different trajectories. This would be observed as refraction.

As described above, this shows an electron taking on the roll of a photon with the exception that it is not necessarily traveling at the speed of light. An electron dislodged while traveling around a nucleus can be used to explain how an electron can travel at such an extremely high speed.

As an electron comes close to a source of time dilation such as a nucleus, the closer time points will move slower than the further causing the electron to spin. Furthermore, as in Figure 10, the plane of the spin would align with the nucleus. As the time points within the electron spin along a plane (s: spin), the motions can be describe based on the direction they are moving relative to the nucleus (u: up and away or d: down and towards)

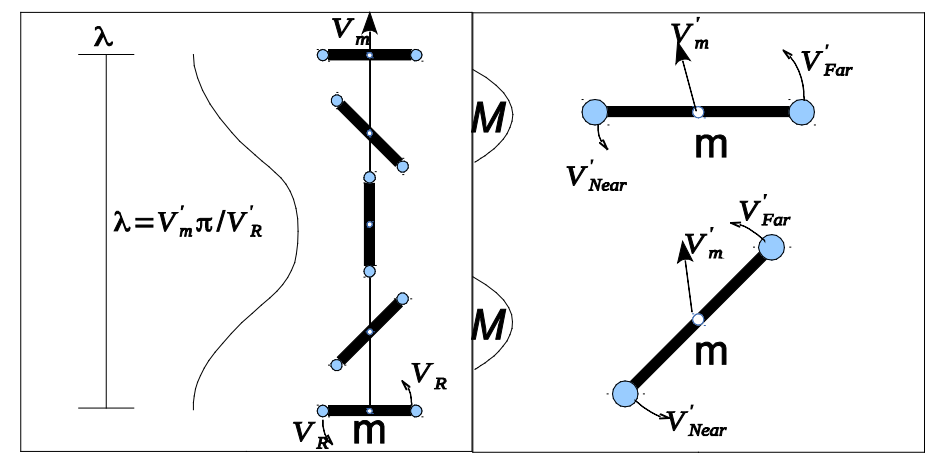

Figure 9. The effects a time dilation has on a rotating electron oscillates as a wave as it travels. As an electron passes close to a mass, it would change direction based on its orientation resulting in a refraction effect. 

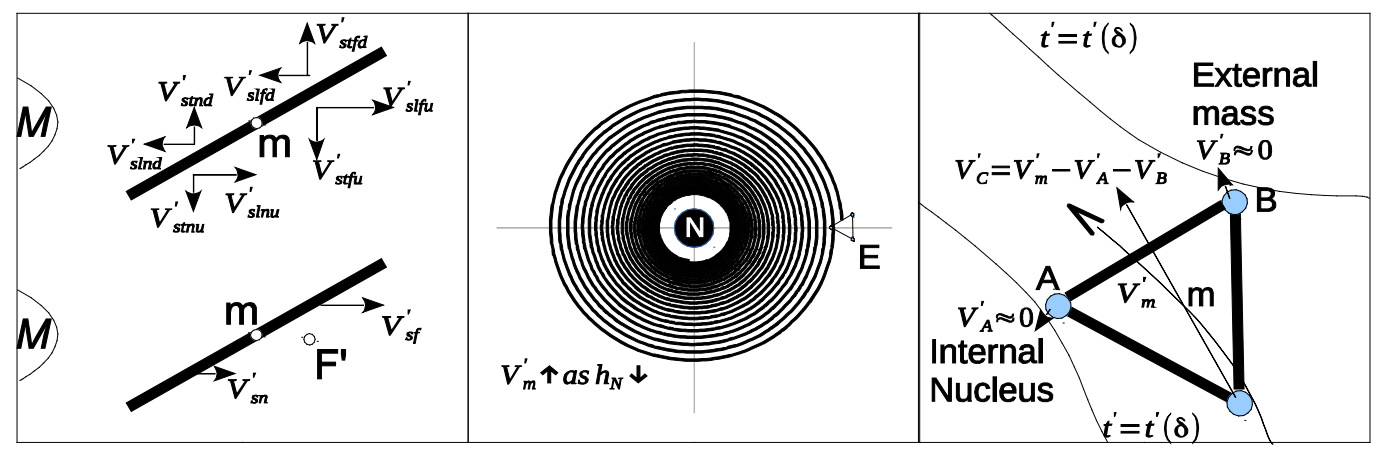

Figure 10. An electron circling a nucleus will rotate to a perpendicular position while its speed increases as it nears the nucleus. As a rotating electron comes into the proximity of another mass it can be expelled at high speeds from the nucleus.

and the position of each time point relative to the mid position of the electron (n: near or f: far). These motions can in-turn be split into the movement in-line with the nucleus and the tangential movement relative to the nucleus (l: in-line or t: tangential).

The tangential movement of a time point in any position as it spins around its mid position in the up direction, will have an equal but opposite tangential movement in the down direction resulting in $V_{\mathrm{stnd}}^{\prime}+V_{\mathrm{stmu}}^{\prime}=0$ and $V_{\text {stfd }}^{\prime}+V_{\text {stfu }}^{\prime}=0$. This leaves the up and down motions combined with the near and far positions to dominate. As the $\partial t^{\prime} / \partial h$ slows time points moving down and towards the nucleus more in the near position than the far and vice versa as they move up and away, $V_{\text {slnd }}^{\prime}+V_{\text {slmu }}^{\prime}<V_{\text {slfd }}^{\prime}+V_{\text {slfu }}^{\prime}$, the focal position, $F^{\prime}$, will be off the plane causing the plane to rotate in the direction of the mass. There will be an unstable saddle equilibrium when the plane of the spin is on the tangent to the nucleus but for any plane not on the tangent, the result will be an equilibrium aligned with the nucleus.

As the electron moves closer to the nucleus, the lateral movement will increase its speed. As described earlier, such lateral motion is a function of how close the electron is to the nucleus, with its motion increasing substantially as it gets closer. As the electron circles the nucleus, it can easily be expelled from an atom at this very high speed. Another set of time dilations, say in the form of another atom, could move into a position that blocks the electron's path. As this occurs, two of the three time points could have time dilate to where it virtually stops while the third continues to travel at this extremely fast speed. This speed would be determined by the speed at which the electron travels around the nucleus while the positions of the time points as it is expelled would determine the rotation.

This is a process that can transform an electron into a photon. As the electron circles around the nucleus we can represent the motion as $V_{m}^{\prime}$. As time points $\mathrm{A}$ and $\mathrm{B}$ move into positions where the time dilations both go to extremes, time point $\mathrm{C}$ would move as $V_{C}^{\prime}=V_{m}^{\prime}$ with the expelled electron $V_{x}$ traveling $V_{C}^{\prime}=V_{x}^{\prime}+V_{C R}^{\prime}+V_{C s}^{\prime}$ where $V_{C R}^{\prime}=V_{R}^{\prime}\left[\partial x_{C} / \partial \theta, \partial y_{C} / \partial \theta, 0\right]^{\mathrm{T}}$ and $V_{C S}^{\prime}=V_{s}^{\prime}\left[\partial x_{C} / \partial \theta, \partial y_{C} / \partial \theta, 0\right]^{\mathrm{T}}$ resulting in $V_{m}^{\prime}=V_{x}^{\prime}+V_{C R}^{\prime}+V_{C S}^{\prime}$ or $V_{x}^{\prime}=V_{m}^{\prime}-V_{C R}^{\prime}-V_{C s}^{\prime}$.

The maximum that the expelled electron, $V_{x}^{\prime}$, can travel will be when $V_{s}^{\prime}$, the spin is stopped and becomes 0 . If we assume that this maximum is the speed of a photon, then the speed of light, $\mathrm{C}$, can be defined as $C=V_{x}^{\prime}-V_{C R}^{\prime}$. As time points $\mathrm{A}$ and $\mathrm{B}$ are stopped, time point $\mathrm{C}$ will rotate around the electron's mid position changing the angles that $\mathrm{A}$ and $\mathrm{B}$ are traveling. When this new angle allows $\mathrm{A}$ and $\mathrm{B}$ to move, the electron would roll off controlling the rotation which, as previously shown, dictates the wavelength.

Since $V_{x}^{\prime}=f\left(V_{m}^{\prime}\right) \quad V_{m}^{\prime}=f(T, \delta)$ then $C=f\left(T, \delta, V_{R}^{\prime}\right)$. This indicates that in a world composed of time dilation, the speed of light is determined by the time dilation coefficient and therefore the time dilation coefficient is not determined by the speed of light. This also connects the speed of light with gravity, since both are the result of the time dilation coefficient and the distance at which the dilated time virtually stops: $C=f(T, \delta)$.

\section{Two Dimensional Electrons: Spin}

As described above, the movement of an electron would also consist of a spin. This is the movement along the plane established by the three time points. In a universe of time dilation, the dynamics of spin also produces peculiar phenomena that explains other behaviors seen in our universe. 
Another interesting characteristic of two dimensional electrons is that they can synchronize their spin. If electrons were to have their planes become parallel, then the spin which one electron has would influence the other's spin. As depicted in Figure 11, time points in each electron would speed up or slow down until they are positioned 60 degrees apart and reach a rate of spin that is the same. A spinning time point that is moving slower than a corresponding time point in the other electron would have its time dilation decrease causing it to speed up. The time points that are spinning faster would approach the corresponding time point in the slower spinning electron causing it to slow. This would continue until they reach the same rate of spin.

Subsequently, other electrons whose planes are parallel would also synchronize their spin and a continuum could result with a large group of electrons spinning in synchronization. This would also allow electrons to become packed among one another. Given the electrons are contained in an overall structure, adding other electrons with a parallel plane would result in more synchronized electrons being suspended by one another with the distances between them reduced. As an electron is added, the resulting $\beta$ of the spin of the nearby electron would move that electron further away. This would bring it closer to the next spinning electron moving it until the distances between all the spinning electrons become the same. More and more electrons could be included within the structure further compacting the electrons.

If the containing medium allows, this process could expand out by taking control of the spin of adjacent electrons and equalizing their distances within the containing medium and the adjacent medium. The adjacent medium thereby becomes a conductor of the spin.

This is what would be observed as electricity in a world of time dilation. As the spin is transferred from the containing medium through a conductor, this spin would be decreased by each slower spinning electron that it encounters. As a static charge is discharged into a ground, the slower spinning electrons in the ground will spin faster while the spin in the static charge will decrease. This happens until there is no longer a definitive spin left in the static charge.

Electrons that are not part of an atom will move about a time dilation universe based on their interactions with other time dilators. This would occur all the time with some electrons moving out of a region and others moving into a region. This movement would not produce the phenomenon that we identify as electricity. The spin is therefore a vital characteristic of electricity in a universe of time dilation.

If electricity were indeed just the movement of electrons, a direct current battery with both terminals connected to a ground, would produce a flow from the terminal with excess electrons to the ground and then from the ground to the terminal with an electron deficit. As in our own universe, this does not happen. A conductor that allows the electrons to synchronize their spin and return the spin back to the battery spinning with the same orientation as it was when it left would complete the required circuit. A battery with terminals connected to a ground would not be able to complete such a circuit and return such a spin back to the battery. This is indicative in what we observe with a direct current.

Furthermore, this compacted spinning collection of electrons would create changes in time dilation which would get amplified by the number of electrons spinning in a linear direction. If the electrons were to spin within a conductor such as a straight wire, they would be arranged in a line. As this occurs they would cause time to dilate more along that line.

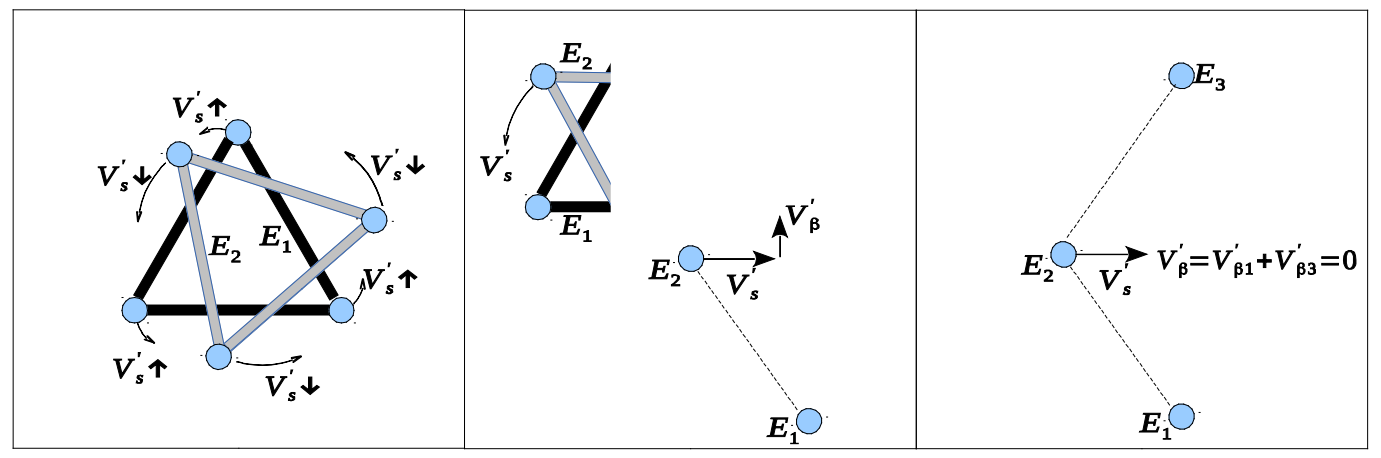

Figure 11. As two electrons spin in the same direction on parallel planes, the slower spinner would speed up while the faster spinner would slow until the two were synchronized. A large group of spinning electrons could become packed. 
As depicted in Figure 12, a line of packed electrons would have an ellipse, $\sum d$. where the sum of the distances to each time point is the same, $k$. The corresponding ellipse, $\sum f(d)$, of equal time dilation, $K$, would extend out further due to the exponential characteristic of time dilation. If we start at a position directly above the mid position of our line of electrons, the distances from each time point would be matched by a corresponding time point on the other side of the mid position resulting in a given time dilation. Moving to a side brings you closer to the time points on that side and further away from time points on the other side. Because the time dilation function is exponential, this additional time dilation caused by the closer side is larger than the reduced time dilation caused by the further side. A greater distance is therefore required to return to the same time dilation.

This additional time dilation along the direction of the line could be defined as the differences between the two ellipses and depend on the number of electrons and how tightly they are packed. Where as in gravity, objects move towards the increased source of time dilation, here, objects move towards this magnified line of time dilation.

This would be perceived as magnetism is perceived in our universe. If we increase the number of lines of electrons, a recognizable pattern emerges. As one moves past the tip of one line towards the second line, the time dilation decreases causing the line of equal time dilation to curve back towards the center of the two lines of electrons. This results in the pattern typically associated with magnetism.

If two separate lines of spinning electrons were placed with their tips near one another, the results would be dependent on the direction of the spin. One could label each tip based on the direction of the spin. As in Figure 13, matching spins would result in opposite tips being placed near one another. As the spin of one line adjusts to match the other, the tangential movement of each spinning time point would move each line towards one another. As in our universe, a universe of time dilations would have opposites attract.

If the spins are opposite one another, the tips coming near are of like labeling. As the lines of electrons spin opposite to one another, the tangential time dilation would combine causing each of the spinning electrons to

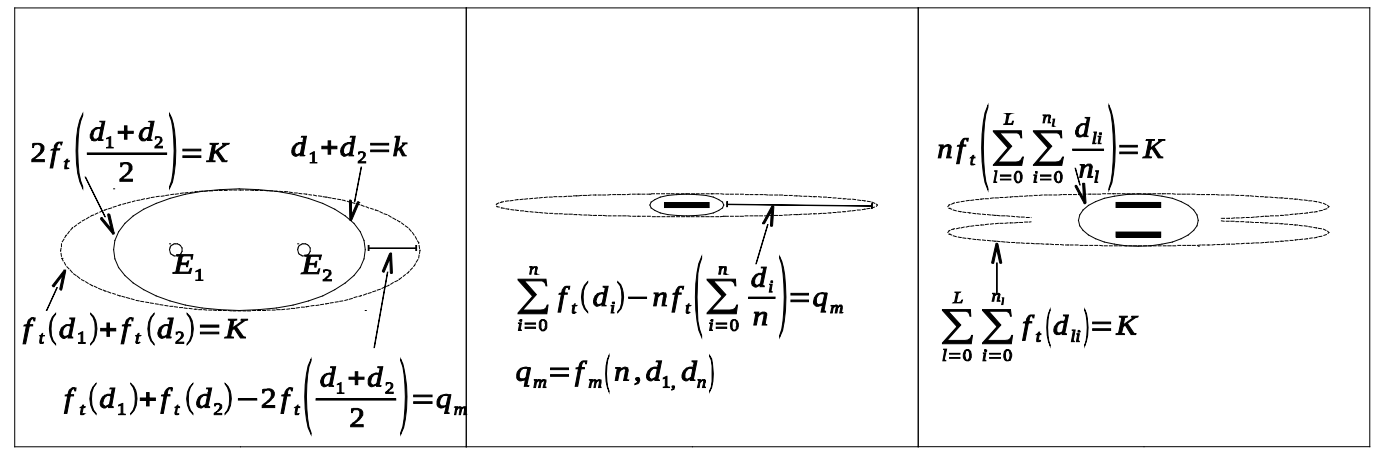

Figure 12. As electrons align and become packed in a straight line, time is dilated greater in the direction of the line leading to an attraction that is dependent on the number of electrons. Multiple lines would result in the pattern typically associated with magnetism.

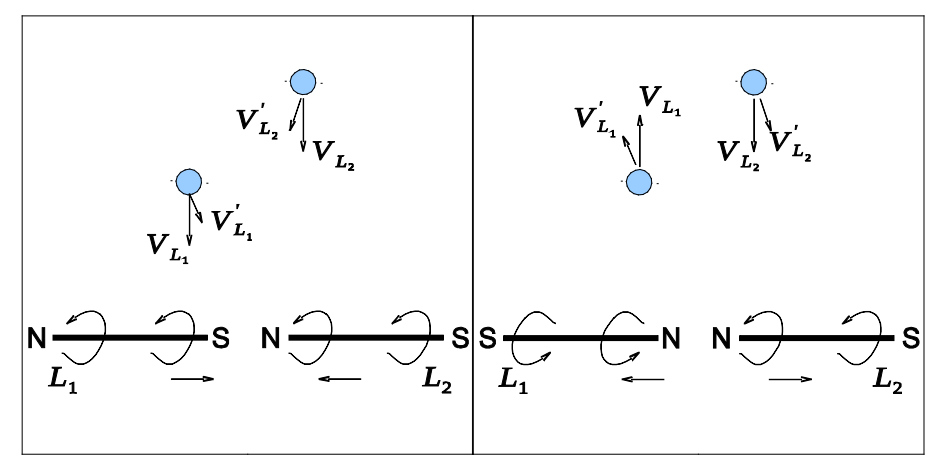

Figure 13. The direction of the electrons' spin would define the polarity of the magnetic field and therefor, the positive/negative aspects of the electricity. 
have a $\beta$ that moves away from the other line. This would cause the two objects containing the spinning electrons to move apart or be repelled. This would occur until the aligned electrons are no longer in a line, the one set of electrons stops spinning, or the two lines of electrons move far enough apart that the total time dilation is no longer big enough to offset the time dilations from other sources.

All together, this explains the phenomena which we see as electricity and magnetism. In a universe composed of time dilation, electricity is caused by the spinning and compaction of electrons while magnetism is the additional time dilation that spinning electrons cause when packed into straight lines. Electricity may require electrons to move to complete a path but the true phenomena is not based solely on such a flow of electrons but the compaction and spin of those electrons. The spin would indicate the speed and the polarity of the electricity or in other words, the voltage while the compaction would determine the magnitude of the electricity or amperage.

Furthermore, in a time dilation universe, it is easy to conceive of scenarios that allow for the transition of one state of an electron to another. Light can interact with collections of time points transforming the electron's rotation to a spin resulting in light transforming into electricity. Likewise, electrons traveling as waves such as light, or spinning as electricity can transition to heat by agitating electrons and atoms causing them to move about at greater and greater rates. Heat and electricity can cause movements that will result in the release of electrons from atoms at high speed resulting in light. These transitions are the same as those transfers of energy that we observe in our universe.

\section{Causality}

The current approach to the issue of causality of much contemporary research is very flawed. When two events are correlated, that is they tend to happen together, researchers will often conclude that the one causes the other. When this is done and the other possibilities of the correlation are ignored, the research is worthless. Not only are such conclusions of little value but they can often be done to get a desired response and mask a less than truthful agenda.

There are several potential explanations when things happen together. The first is that they may be just a coincidence. This can be further exaggerated when the occurrence of the two events leads to their detection. The spilling of gasoline and the lighting of a match may be separate random events and may go unnoticed. However, when they happen together, they are quite noticeable. Using only this observed correlation, one could incorrectly conclude that spilling gasoline causes the match to light. When observing such a correlation we must consider the potential that there is no causal relationship between the two events.

The second flaw is that of the direction of the causality. When confronted with a strong correlation between event A and event B, it is a mistake to conclude that A causes B. We must examine the other possibilities including that B causes A. A university recently touted the correlation between good grades and taking a full 15 credits of classes and, with their obvious agenda, indicated that students should take 15 credits so they will also get good grades. They neglected to examine the possibility that students that are struggling and getting bad grades are more likely to cut back on the number of classes they are taking. In this case, it is more logical to expect poor grades to lead to a lighter course load. Here, they incorrectly conclude that A causes B, when the more logical conclusion is that $\mathrm{B}$ causes $\mathrm{A}$.

A third possibility is that both A and B are caused by a third event C. When a game of billiards is started one may observe the eight ball moving at the same time as the fifteen ball. They are correlated. It is incorrect, however, to conclude that the eight ball causes the fifteen ball to move or that the fifteen causes the eight to move. Instead, the true causality is that the cue ball is causing both to move. Looking for such a third party is often overlooked in poor research and the research ends up making an incorrect conclusion.

To complicate things further, the events may not be causal at all. A could be a catalyst that allows B to cause C. Leaving the corral gate open does not cause the cows to leave but merely allows it to happen. The cows could be startled by a separate event and with the open gate, they would be able to leave.

Contemporary thoughts in Physics have failed in this area, too. The focus has been that moving objects cause time to dilate. Time dilation has been associated with movement and it has been concluded that this movement causes the time dilation. Other possibilities have failed to be examined even though there is not a good explanation as to how movement causes this time dilation. In a universe made up of time dilating points, movement does not cause time to dilate relative to other objects, but time dilation must occur for objects to move.

In a time dilation world, two bodies that exist near one another will continue to exist near one another until 
the relative time of one becomes different from the other. To utilize the twin example [2], for the one twin to move away from the other, his relative time must change either by being pulled as in gravity or compressed as in being pushed. If relative time were to remain the same for both twins, they would remain together. To make the one twin be propelled away at the speed of light, his relative time must be made to come close to stopping. Therefore propulsion in such a universe is fundamentally a method of changing relative time in order for objects to move apart. In such a universe, the twin, that feels a $\mathrm{G}$ force upon takeoff, would actually be feeling the time points in their body becoming closer, thus dilating time.

\section{Time Dilation Coefficient}

Until now, I have purposely neglected to further define the time dilation coefficient, $T$. Contemporary views places this coefficient at the speed of light squared. If this is the case then there is width to a time point in that the time dilation would approach infinity as $h$ approaches $1 / C^{2}$. This distance would also present itself in the wave pattern of light, however, it may be too small for it to be measured in either situation. I have already shown that in a universe made up of time dilating points, the speed of light is determined by $T$ and $\delta$, the distance at which time essentially stops, but there is no obvious tie to the square root of $T$.

Other possibilities do exist though. $T$ could be infinity resulting in the time point truly being a point. Here, the time dilation would approach infinity as $h$ approaches 0 . Under these circumstances the speed of light squared would just be a really good approximation for infinity. The differences between the speed of light squared and infinity may be hard to distinguish in the common occurrences in a universe.

Yet another intriguing possibility is that $T$ is not a constant at all. It could be a value that has been expanding since time began. Under this possibility, the constant would be the beginning of a universe, "The Big Bang” [3], and $T$ could be a function of the total amount of time that has accumulated. Under this scenario, $T$ would be forever approaching infinity and perhaps the speed of light squared is an approximation of the amount of time that has passed since the beginning.

It is possible that there are no constants in the world and it is only human nature that tries to identify a constant when we are confronted with a result. Other values of $T$ may also be valid but any large number would drive the described relationships within a universe of time dilation in a similar fashion.

\section{Conclusion}

In that a universe made up of only time dilation behaves identical to ours, one can conclude that our universe is actually made up of only time dilation. Movable points which dilate the time of other movable points, time points, make up the basis for our universe. These time points come together to form the various subatomic and atomic components of our world. These structures form equilibriums that in-turn move about with the structure's individual time points moving together but under different relative time. Relationships that are thought to be forces are actually phenomena associated with the movement and interactions of these time points. Gravity, atomic repulsion, and magnetism are specific behaviors of moving time points. Light and electricity are specific behaviors of electrons and their two-dimensional structure. In all, it appears that we are in a universe made up of relative time. Time is not only relative, but relative time is everything.

\section{References}

[1] Collier, P. (2012) A Most Incomprehensible Thing: Notes towards a Very Gental Introduction to the Mathematic of Relativity. Incomprehensible Books.

[2] Von Laue, M. (1911) Physikalische Zeitschrift, 13, Article ID: 118120.

[3] Singh, S. (2004) Big Bang: The Origin of the Universe. Harper Collins Publisher, New York. 